\title{
Outcome of Gunshot Abdominal Injuries
}

\author{
Neville Mengjo Bonko, ${ }^{1,2}$ Maryam Badawy, ${ }^{1,2}$ Keafon Nchifor, ${ }^{1,2}$ Mina Mumba, ${ }^{1,2}$ Leon Omboga, ${ }^{1,2}$ Robert \\ Mugo, ${ }^{1,2}$ Sandra Kimani, ${ }^{1,2}$ Catherine Kwamboka, ${ }^{1,2}$ Moce Moraa, ${ }^{1,2}$ Akem Dimala, ${ }^{4}$ Joseph Githaiga, ${ }^{1,2,3}$ \\ ${ }^{1}$ Department of Surgery, University of Nairobi, Kenya \\ ${ }^{2}$ Kenyatta National Hospital, Kenya \\ ${ }^{3}$ Nairobi Surgical Skills Center, Kenya \\ ${ }^{4}$ Bloomberg School of Public Health
}

Correspondence to: Dr. Bonko Neville, PO Box 30197-00100, Nairobi; email: nevillemenchs@gmail.com

\begin{abstract}
Aim: To describe prevalence, management and factors determining outcomes in patients presenting with gunshot abdominal injuries. Method: We retrospectively analysed all cases of gunshot to the abdomen received at Kenyatta National Hospital from October 2013 to October 2017. Patients' demographic and clinical data were collected from their case notes. Data analysis used Fisher's exact test and binary logistic regression. A p-value of $<0.05$ was considered statistically significant. Results: A total of 1,588 records of patients with abdominal injuries were analysed. Of these, 209(15.3\%) were cases of gunshot to the abdomen. The mean age was 31.5 years; male to female ratio was 8:1. Exploratory laparotomy was the preferred management in 161(77\%) patients, selective nonoperative management in 11(5.3). Negative laparotomy rate was $8.7 \%$, inpatient mortality $20 \%$ and complication
\end{abstract}

\section{Introduction}

Injuries caused by violence and accidents constitute the second most common cause of mortality worldwide (1). These injuries are a public health challenge especially in resource-limited settings. The abdomen is one of the most commonly injured part of the body; approximately $90 \%$ of the mortality related to penetrating abdominal injuries are attributable to gunshot wounds (2). Gunshot abdominal injuries (GAI) are potentially fatal due to the risk of hemorrhage and visceral injury.

Traditionally, laparotomy has been mandatory to manage all abdominal gunshot wounds. As much as this is effective in identifying and managing all possible injuries, mandatory laparotomy has been shown to have a complication rate of $15-50 \%$ with a prolonged hospital stay (3). With advancements in imaging techniques and increased understanding of possible extent of injury, there has been a shift towards selective non-operative management (4). Initial evaluation of the patient presenting with an abdominal gunshot wound is rate $26 \%$. Age, time from injury to admission, assisted breathing on admission, need for transfusion, and number of complications independently predicted mortality. Conclusion: Gunshot abdominal injuries are commonly encountered at our setting and these are associated with significant mortality and development of complications. With careful selection, some patients can be successfully managed non-operatively.

Keywords: Gunshot, Abdomen, Mortality, Outcome Ann Afr Surg. 2020; 17(1):30-34.

DOI: http://dx.doi.org/10.4314/aas.v17i1.8

Conflicts of Interest: None

Funding: None

(C) 2020 Author. This work is licensed under the Creative Commons Attribution 4.0 International License.

therefore critical in deciding the management options for the patient. To the best of our knowledge, no studies in our setting have specifically addressed gunshot injuries to the abdomen. In this study, we sought to describe the prevalence, management and factors determining outcomes of gunshot abdominal injuries in our setting.

\section{Materials and Methods}

In this retrospective study, we analyzed all cases of gunshot to the abdomen received at the Kenyatta National Hospital-Kenya's largest referral hospital and the seat of medical and surgical training - from October 2013 to October 2017. The accident and emergency unit receives approximately 250 to 300 patients a day most of whom are trauma patients. The team within the trauma bay comprises experts in triaging, resuscitation, medical examination and assessment, and referral to appropriate firms for definitive management. This study was approved by the University of Nairobi/KNH Ethics and Research Committee (Protocol number: P233/05/2017). Our 
data collection tool was designed to capture data on the following variables: site of abdominal injury(ies), number of abdominal organs injured, extent of injury to the organs injured, extra-abdominal associated injuries, treatment approach, length of hospitalization, and treatment outcome. Medical records of patients with abdominal injuries not due to gunshot and those that did not contain all the information as per our data collection tool were excluded. Figure 1 summarizes the management protocol for our study setting.

We defined superficial abdominal injuries as those with no evidence of peritoneal breach either on physical examination or by imaging. Injury severity was quantified using the injury severity score (ISS) and penetrating abdominal trauma index (PATI) score (5). We defined poor outcome as death or development of complication either related to the injury, its management or both. These complications included intra-abdominal bleeding, abdominal compartment syndrome, anastomotic leak, abdominal abscess, surgical site infection, septic shock, deep venous thrombosis, pulmonary embolism and urinary tract infection. The anterior abdomen is defined as the area between the xiphoid and costal margins superiorly, the pubic symphysis and the inguinal ligaments inferiorly, and the midaxillary lines posteriorly. Laparotomy was negative when no injuries to abdominal organs were found. Data was keyed into SPSS version 22 for analysis. Descriptive statistics such as means, medians and percentages were used in data analysis. Fisher's exact test was used to establish associations between independent variables and outcomes. Binary logistic regression analysis was performed using a forward stepwise selection method based on likelihood ratios. A p-value of $<0.05$ was considered significant.

\section{Results}

Out of 1,588 patients with abdominal injuries requiring admission, gunshots were responsible for $15.3 \%$ (209) of cases - 186 males and 23 females_, giving a male:female ratio of 8: 1 . Most patients - 139 (66.5\%) were in the second and third decades of life. The mean age was 32 years $+/$ - 10.7. At the time of presentation, $139(66.5 \%)$ were hemodynamically normal. Of the hemodynamically abnormal patients, 19(9\%) had signs of hypovolemic shock (SBP $\leq 100 \mathrm{mmHg}$ and pulse $\geq 100$ beats/min). Hemodynamically abnormal patients
Indications for immediate laparotomy present?

1. Peritonism - abdominal tenderness, rebound, guarding, rigidity

2. High spinal cord injury preventing abdominal examination 3. Haemodynamic instability 4. Evisceration

5. Equivocal CT and FAST, abdominal X-ray results

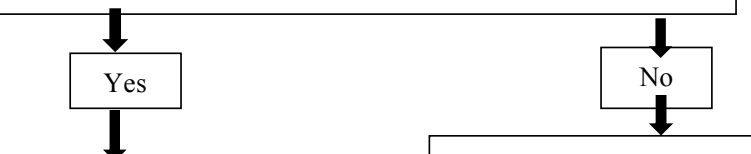

Immediate laparotomy

Peritoneal penetration identified? Investigations include:

1. FAST

2. Plain Abdominal X-ray 3. $\mathrm{CT}$

Laparotomy for extensive internal organ damage or at surgeon's discretion OR

Selective non-operative managementwith serial physical examinations and close monitoring for minor internal organ injuries in patients who remain hemodynamically stable or at surgeon's discretion.

Figure 1. Summary of the management protocol for gunshot abdominal injuries. (23)

were significantly more likely to develop complications $(\mathrm{p}=0.001)$ and die $(\mathrm{p}=0.025)$ than hemodynamically normal patients. About one-half of the population (54\%) needed transfusion on arrival, with an average of 2 units of blood required per patient. Mean duration from injury to commencement of treatment was 10 hours and ranged from 30 minutes to 121 hours (Table 1). Risk of mortality was directly associated with increasing ISS. Mortalities of 23\%, 67\% and $100 \%$ were recorded for ISS of $<25,26-50$, and $>50$ respectively (Table 2 ). High penetrating abdominal trauma index scores significantly correlated with mortality and likelihood for development of complications (Table 2). Most injuries (67\%) were localized to the anterior abdomen, with the rest occurring in the thoraco-abdominal region (21\%), transpelvic (9\%), gluteal (3\%) and the back (1\%). Diagnostic investigations were performed in $81.3 \%$ of patients: of these 42\% (71) had FAST, 29\% (50) had abdominal CT scan, 26\% (44) had plain abdominal x-ray, 2\% (4) had DPL and $0.6 \%$ (1) had MRI. Laparotomy was the preferred management in $161(77 \%)$ patients, selective non-operative management in $11(5 \%)$ and surgical debridement for superficial wounds in 33(15.8\%).

Delayed laparotomy was performed in 2 out of the 11 patients selected for non-operative management. 
Table 1. Patients' demographic and clinical characteristics at presentation

\begin{tabular}{|c|c|c|c|}
\hline $\begin{array}{l}\text { Clinical } \\
\text { characteristics at } \\
\text { presentation }\end{array}$ & Frequency & $\begin{array}{l}\text { Developed } \\
\text { complication }\end{array}$ & Died \\
\hline \multicolumn{4}{|l|}{ Age } \\
\hline$<10$ & 1 & 0 & 0 \\
\hline $10-20$ & 30 & 8 & 7 \\
\hline $21-30$ & 74 & 23 & 14 \\
\hline $31-40$ & 65 & 12 & 9 \\
\hline $41-50$ & 27 & 9 & 5 \\
\hline $51-60$ & 10 & 2 & 6 \\
\hline $61-70$ & 2 & 1 & 1 \\
\hline \multicolumn{4}{|l|}{ Gender } \\
\hline Male & 186 & 49 & 35 \\
\hline Female & 23 & 6 & 7 \\
\hline \multicolumn{4}{|c|}{ Systolic BP $(\mathrm{mmHg})$} \\
\hline$<100$ & 42 & 12 & 12 \\
\hline$>100$ & 167 & 43 & 30 \\
\hline \multicolumn{4}{|l|}{ Pulse rate } \\
\hline$<100$ & 67 & 29 & 17 \\
\hline$>100$ & 142 & 26 & 25 \\
\hline \multicolumn{4}{|l|}{ Breathing } \\
\hline Assisted & 56 & 31 & 33 \\
\hline Self-breathing & 144 & 19 & 9 \\
\hline \multicolumn{4}{|c|}{ Need for transfusion } \\
\hline Yes & 113 & 44 & 37 \\
\hline No & 93 & 8 & 4 \\
\hline \multicolumn{4}{|c|}{ Duration (hours) to presentation } \\
\hline$<6$ & 77 & 29 & 12 \\
\hline $6-12$ & 41 & 12 & 9 \\
\hline $12-24$ & 14 & 4 & 5 \\
\hline$>24$ & 8 & 1 & 2 \\
\hline
\end{tabular}

Negative laparotomy rate was $8.7 \%$. Mean number of injured organs per patient was $3+/-1.37$, and the ileum was the most frequently injured organ in $67(18 \%)$ patients (Fig. 2).

Table 2. Relationship between trauma scores and outcomes

\begin{tabular}{cccc}
\hline $\begin{array}{l}\text { Trauma / } \\
\text { Score ranges }\end{array}$ & Frequency & $\begin{array}{l}\text { Develop } \\
\text { complications (\%) }\end{array}$ & Died (\%) \\
\hline Injury severity score (ISS) & & \\
$<25$ & 177 & $41(23)$ & $33(18.6)$ \\
$26-50$ & 20 & $8(40)$ & $8(40)$ \\
$>50$ & 1 & $1(100)$ & $1(100)$ \\
Penetrating abdominal trauma index & $(P A T I)$ & \\
$<25$ & 171 & $36(21)$ & $28(16.4)$ \\
$26-50$ & 29 & $15(52)$ & $14(48.3)$ \\
$>50$ & 0 & 0 & 0 \\
\hline
\end{tabular}

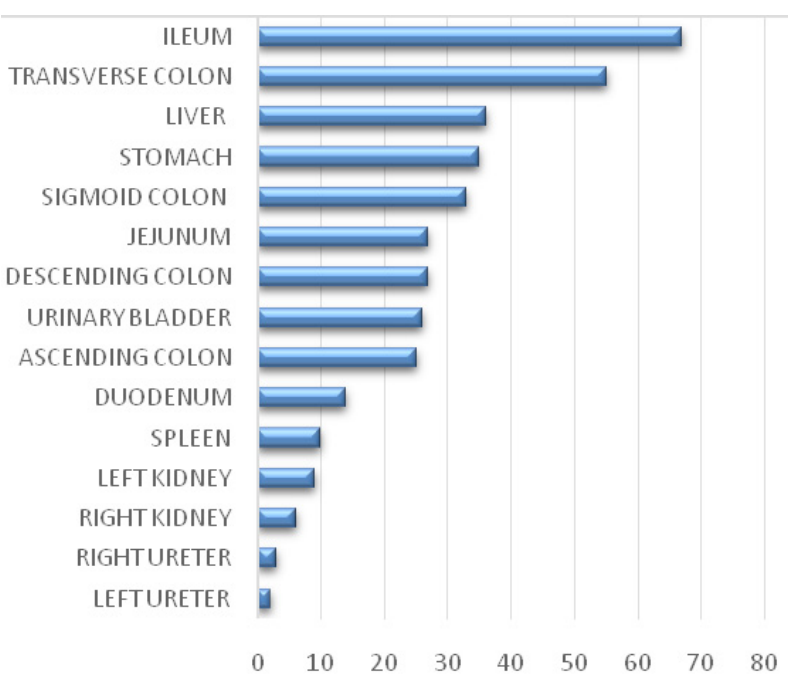

Figure 2. Frequency (\%) of intra-abdominal injuries.

The study population had an overall $26 \%$ complication rate; the most frequently encountered complication was intra-abdominal bleeding. Four patients (2\%) died on arrival, and inpatient mortality was $20 \%$ (42). Median length of hospital stay was 10 days and ranged from 1 to 194 days. Logistic regression analysis showed a significant influence of age, need for transfusion, number of complications, duration from injury to presentation and assisted breathing (on admission) on mortality of patients $(\chi 2(6)=66.04, \mathrm{p}=0.001)$. The model explained $61.3 \%$ variance (Nagelkerke) and was able to identify $87 \%$ of cases successfully with a sensitivity and specificity of $91.3 \%$ and $71.4 \%$ respectively.

\section{Discussion}

Abdominal injuries constitute a global public health burden and present diagnostic, therapeutic and prognostic challenges to healthcare professionals. These injuries have been described as a disease in evolution (6). Our main objective was to characterize GAI for their prevalence, pattern, management and outcomes. Similar to other penetrating abdominal injuries, GAI is a predominantly male entity, affecting mostly young adults in their second and third decades of life $(7,8)$. Adults in their third decade are in the active occupational age bracket and are more likely to be involved in gun violence by assault (1). Studies on penetrating abdominal injuries, including those previously done in our setting, reported higher proportions of gunshot to the abdomen than found in our study $(8,9)$. These studies were mostly prospective studies with small sample sizes, or studies in which abdominal injuries of different etiologies were considered. 
The prevalence is likely to be lower if all forms of abdominal injuries are studied as we did in our setting. Musau et al. (7) in his study in the same setting, found a prevalence of $21.3 \%$. This difference could be because of the short duration ( 1 year) of the study and small sample size ( 80 patients).

The anterior abdomen was the most frequently injured in our population. This was similarly reported in other studies $(10,11)$. Thoraco-abdominal injuries were the second most common (19\%) injuries. Salim et al. had a similar proportion (20.1\%) in his study that exclusively analyzed gunshots to the abdomen, as in our case (11). Maha et al. (10) looking at other penetrating abdominal injuries found a lower proportion (8.2\%). Back injuries were least common. These were comparatively lower than the $14.5 \%$ found by Salim et al. but similar to Maha et al. findings $(10,11)$. However variable the site of injury may be from study to study, the anterior abdomen is most frequently injured, and the back is the least injured in gunshots to the abdomen.

Increasing duration from injury to commencement of treatment was significantly associated with mortality but did not influence the development of complications in our study. As we have shown, patients with less severe injuries were less likely to develop complications. These patients tend to present to hospital later than those with severe injuries.

A smaller proportion of patients in our study had hypovolemic shock (9\%) than reported by other studies. Patients with gunshot wounds are more likely to present early, before vital signs start deteriorating (7). Hemodynamic stability nonetheless does not exclude the need for emergency intervention in this group of patients as instability may develop variably over a few hours. If the decision has been made to avoid emergency laparotomy, the key is close monitoring with serial physical examination (12). Patients with hemodynamic instability, however, were more likely to develop complications and die than the rest of the population, as similarly reported by Iflazoglu et al. (13). Hemodynamic status determines the need for investigations in patients with GAI as with other injuries. Patients with hemodynamic instability or frank peritonitis require emergency laparotomy, usually without need for investigations. Stable patients will need investigations and serial monitoring and may even benefit from selective non-operative treatment (12). The finding that $66.5 \%$ of the population in this study were hemodynamically normal but $81.3 \%$ had diagnostic investigations may be explained by the presence of readily available investigations in Kenyatta National Hospital or may suggest that a fraction of our patients was overinvestigated. Thus, thorough clinical assessment should form the basis for decision on investigation of patients with abdominal injuries (14). Diagnostic peritoneal lavage (DPL) and FAST are widely used in other centers. Githaiga and Adwork showed that DPL is an easy-toperform, cheap and reliable assessment tool in patients with (blunt and penetrating) abdominal trauma (15). However, other centers hold that this investigation plays no role in penetrating trauma, and the place of FAST in penetrating trauma is still unknown. Helical CT has been shown to be superior to FAST in excluding peritoneal penetration in patients with gunshot abdominal injuries $(12,16)$. In our study however, more patients had a FAST than CT scan. This is because FAST is relatively affordable. CT scans were mostly performed for patients in whom FAST was inconclusive in deciding on the next step in the management. This is not standard practice and calls for revision of management protocols in our setting. None of our patients had abdominal laparoscopy, because it was unavailable at the time of this study.

As the management of abdominal injuries continues to evolve, routine laparotomy for all penetrating injuries has been dichotomized to involve selective nonoperative management though with variable degrees of success. Management of penetrating abdominal injuries by compulsory laparotomy has been disputed by other studies on grounds that negative laparotomies not only lead to increased cost and prolong hospital stay but also lead to high re-operation rates $(17,18)$. Gunshot abdominal injuries have been managed successfully by selective non-operative management with minimal complication rates (11). We found a low negative laparotomy rate $(8.7 \%)$ comparable to those in other settings $(13,19)$. Musau et al. however had a higher negative laparotomy rate $(16.1 \%)$ in the same setting (9). This might be due to the mixed injury population and varied treatment approaches by different surgical firms in his study, but also it might be an indication of improvement in management of patients with abdominal injuries in our setting.

Management of gunshot injuries is associated with high complication rates because of severity of injury attributable to missile characteristics (13). Due to high kinetic energy, explosive bullet particles injure not only the organs they enter but also the surrounding tissues. Furthermore, when bones are involved, broken pieces can injure other organs. Missed injuries are a common cause of postoperative complications (20). We found a 
higher complication rate (26\%) than previously reported for mixed abdominal injuries (21). Higher complication rates have been reported in other studies (22). This difference likely arises from the type of firearms used in different settings. The ileum and colon were the most commonly injured in our study, and these organs have been shown to be the origin of septic complications following abdominal injuries (19). However, the most frequently encountered complication in our setting was intra-operative and postoperative bleeding, as also reported by Lap et al. (22). Mortality has been shown to be generally higher with GAI than with other causes of abdominal injuries (19). Factors that independently predicted mortality in our setting were age, increasing duration from injury to presentation at hospital, assisted breathing on admission, need for transfusion and number of complications.

A limitation is that this was a retrospective study and inaccurate entries in patients' case notes could not be verified. Also, we could not classify firearms into high and low velocity categories as these were not consistently documented in case notes.

In conclusion, gunshot to the abdomen is common in our setting. These injuries are associated with significant development of complications and mortality. With careful selection, some patients can be successfully managed non-operatively. We recommend strict adherence to surgical protocols, timely intervention, proper triage and management to avert mortality and morbidity.

\section{Acknowledgments}

We heartily thank the workers of the records department of Kenyatta National Hospital for cooperating with us in providing the data we needed for this study.

\section{References}

1. Hoffmann C, Goudard Y, Falzone E, et al. Management of penetrating abdominal trauma: What we need to know? Ann Fr Anesth Reanim. 2013; 32(2):104-11.

2. Butt M, Zacharias N, Velmahos G. Penetrating abdominal injuries: Management controversies. Scand J Trauma Resusc Emerg Med. 2009; 17:19.

3. Sosa J, Baker M, Puente I, et al. Negative laparotomy in abdominal gunshot wounds: Potential impact of laparoscopy. J Trauma. 1995; 38(2):194-7.

4. Velmahos G, Demetriades D, Toutouzas K, et al. Selective non-operative management in 1,856 patients with abdominal gunshot wounds: Should routine laparotomy still be the standard of care? Ann Surg. 2001; 234(3):395-402.

5. Sikic N, Korac Z, Krajacic I, et al. War abdominal trauma: Usefulness of penetrating abdominal trauma index, injury severity score, and number of injured abdominal organs as predictive factors. Mil Med. 2001; 166(3):226-30.

6. Smith J, Caldwell E, D’Amours S, et al. Abdominal trauma: A disease in evolution. ANZ J Surg. 2005; 75(9):790-4.

7. Musau P, Jani P, Owillah F. Pattern and outcome of abdominal injuries at Kenyatta National Hospital, Nairobi. East Afr Med J. 2006; 83(1):37-43.

8. Edino S. Pattern of abdominal injuries in Aminu Kano Teaching Hospital, Kano. The Niger Postgrad Med J. 2003; 10(1):56-9.

9. Osime C, Oludiran O. Penetrating abdominal injury cases admitted in University of Benin Teaching Hospital. Annals of Biomedical Science. 2004; 3:39-44.

10. Omer M, Hamza A, Musa MT. Penetrating abdominal injuries: Pattern and outcome of management in Khartoum. IJCM. 2014; 5(1):18-22.

11. Salim A, Velmahos G. When to operate on abdominal gunshot wounds. Scand J Surg. 2002; 91(1):62-6.

12. Lamb C, Garner J. Selective non-operative management of civilian gunshot wounds to the abdomen: A systematic review of the evidence. Injury. 2014; 45(4):659-66.

13. Iflazoglu $\mathrm{N}$, Ureyen $\mathrm{O}$, Oner OZ, et al. Complications and risk factors for mortality in penetrating abdominal firearm injuries: Analysis of 120 cases. Int J Clin Exp Med. 2015; $8(4): 6154-62$.

14. Mnguni M, Muckart D, Madiba T. Abdominal trauma in Durban, South Africa: Factors influencing outcome. Int Surg. 2012; 97(2):161-8.

15. Githaiga J, Adwok J. Diagnostic peritoneal lavage in the evaluation of abdominal trauma using the dipstick. East Afr Med J. 2002; 79(9):457-60.

16. Ginzburg E, Carrillo E, Kopelman T, et al. The role of computed tomography in selective management of gunshot wounds to the abdomen and flank. J Trauma. 1998; 45(6):1005-9.

17. Peev M, Chang Y, King DR, et al. Delayed laparotomy after selective non-operative management of penetrating abdominal injuries. World J Surg. 2015; 39(2):380-6.

18. Renz B, Feliciano D. The length of hospital stay after an unnecessary laparotomy for trauma: A prospective study. J Trauma. 1996; 40(2):187-90.

19. Saghafinia M, Nafissi N, Motamedi M, et al. Assessment and outcome of 496 penetrating gastrointestinal warfare injuries. J R Army Med Corps. 2010; 156(1):25-7.

20. Adeyinka A, Yunusa T, Nonye E. Profile of abdominal trauma in Federal Teaching Hospital, Gombe, North-East, Nigeria: A cross-sectional study. IJMSIR. 2015; 4:41-5.

21. Celen O, Oguz S, Dogan M. Abdominal gunshot wounds: Retrospective analysis of 164 patients. Ulus Travma Derg. 2001; 7(4):258-61.

22. Tap H, Mesci A, Eryýlmaz M, et al. The affecting factors on the complication ratio in abdominal gunshot wounds. Ulus Travma Acil Cerrahi Derg. 2011; 17(5):450-4.

23. Christopher C, Ernest E. Initial evaluation and management of abdominal gunshot wounds in adults: UptoDate; 2018. Available from: https://www.uptodate.com/contents/ initial-evaluation-and-management-of-abdominal-gunshotwounds-in-adults? source $=$ autocomplete\&index $=0 \sim 1 \&$ sear $\underline{\mathrm{ch}=\text { gunshot }}$ 